\title{
Formation Control of Satellites in Low Earth Orbit by Using Moving Masses
}

\author{
Jianqing $\mathrm{Li}^{*}$, Shameng Wen ${ }^{\dagger}$ and Hua Zhong $\ddagger$ \\ Hangzhou Dianzi University, Hangzhou, 310018, China
}

\begin{abstract}
This paper investigates a formation control technique based on the use of moving masses. First, the mechanism of the moving mass control is conducted to reveal the relation between the attitude and the offsets of moving masses. Then, to achieve the desired formation control, the aerodynamic force generated by the change of attitudes is used as the control input to implement the orbit control. The moving masses and magnetic torquers constitute a combined actuator to drive the satellite attitude. To deal with the offset saturation of moving masses, an adaptive controller is investigated. Finally, a simulation on two satellites formation is provided, demonstrating the feasibility of the proposed method.
\end{abstract}

\section{Introduction}

Formation flying is a powerful technology for many space missions such as communication, observation, and environment measurement. In particular, the formation at low-Earth orbit (LEO) has been considered seriously for applications due to its wide coverage and low cost. One of the critical technology in satellite formation is the relative motion and attitude control. At the LEO altitudes, the atmospheric forces on satellite provide a passive way for the attitude and relative motion control. This potential control technique shows fuel savings and low cost, which especially on small satellite. The use of the aerodynamic force is easy to implement by using flat plates [1], in which the aerodynamic drag is mainly applied to realize the minimum time rendezvous [2], formation keeping [3], and attitude maneuvers[4]. Although the aerodynamic lift were negligible in most applications, it still can be used for orbital transfer at very low altitudes[5].

Moving mass control is another possible way to perform satellite attitude and orbit control. In the earlier study, the moving mass control is used to deal with the attitude stabilization in terms of environmental disturbance. For example, a moving mass attached on the satellite be served as a balance device to reduce the attitude oscillation. The moving mass is only $1 \%$ of the total mass and reduce the fuel consumption. Another application of moving masses is to deal with some undesired dynamical behaviors. By using one moving mass and one spring, [6] proposed to remove the phenomenon of nutation which appears in the spinning spacecraft. [7] design two orthogonal moving masses to

\footnotetext{
*Professor, College of Communication Engineering.

$\dagger$ Associate professor, College of Communication Engineering,wsm_hdu@163.com.

†Professor, College of Communication Engineering.
} 
implement the spinning stabilization. These moving mass configurations make spacecrafts have ability to eliminate the coning motion. In addition, the moving mass are used to assist solar-sails for the pitch and yaw control[8], and to change the center of mass for propulsion device [9]. It shows that the use of moving masses as auxiliary control are mainly applied to help detumble spacecraft or complete the traditional attitude actuators.

With the development of the LEO satellite system, the use of atmospheric forces with moving mass control has been proposed and applied for attitude maneuvering. The key concept of using moving masses based on atmospheric forces is to modify the distance between the satellite's center of pressure and the center of mass by shifting the internal mass. This control technique results in a change of the aerodynamic torque generated from the aerodynamic drag. Chesi [10] proposed a particular moving mass configuration that using the real-time relocation of the center of mass to the center of rotation to balance spacecraft simulators. The experiment shows that the automatic balancing system is suitable for small-size satellite simulators. Based on Chesi's concept, a typical engineering design was proposed as the "Shift-Mass Sat" 3U CubeSat with three orthogonal shifting masses [11]. The proposed moving mass configuration focuses on the rejection of aerodynamic disturbances in the very low Earth orbit, in which the parameters of moving masses (offsets and mass ratio) are considered in practice. [12] investigated two combined actuators (a reaction wheel or magnetic torquers) with moving masses to achieve higher accuracy at LEO. The results shows that the use of three moving masses and three magnetic torquers not only implement attitude maneuvering but greatly decrease the residual oscillation error. [13] explored the mechanism for precision formation keeping with moving masses. The magnitude relationship between the relative position and the offsets is determined by simplifying the nonlinear influence of the relative motion.

To the authors' knowledge, the existing research mainly concerns the moving mass control system design, the control ability and stability are less studied. In this work, we focus on the dynamic analysis and formation control of LEO satellites with internal moving masses and magnetic torquers. Firstly, the control relation between the attitude and the offsets of moving masses are conducted to achieve the attitude stability condition. By analyzing the mechanism of the moving mass control, the control capacity and maximum offsets of moving masses are determined. Then, considering that the coupling between the orbit and attitude dynamics in the aerodynamic disturbance. An optimal control law based on Nash strategy is investigated to achieve the desired formation. Then, in order to handle the offset saturation of moving masses, an adaptive attitude control method and the allocation of actuators are investigated. Finally, the simulation is carried out to verify the proposed formation control approach.

\section{Problem formulation}

\section{A. Configuration of Moving Mass Satellite}

We describe one design of the mechanism described in Fig.1. The satellite body is equipped with the three moving masses along three orthogonal axes in the body-fixed coordinate system $o_{b}-x_{b} y_{b} z_{b}$, where $o_{b}$ is the center of mass of 
the satellite body. Each moving mass can be driven to change the center of mass of whole system $o_{s}$.

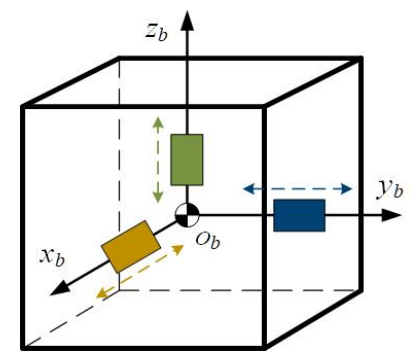

Fig. 1 Schematics of the cubic satellite with the moving masses

Let $\boldsymbol{r}_{i}, \boldsymbol{r}_{b}$, and $\boldsymbol{r}_{s}$ be the position vector of the $i$ th moving mass, the center of mass of the satellite body, and the center of mass the whole system with respect to $o_{b}$. Then, we have

$$
\boldsymbol{r}_{s}=\boldsymbol{r}_{b}+\sum_{i=1}^{3} \mu_{i} \boldsymbol{r}_{i}
$$

where $\mu_{i}$ is the mass ratio defined as

$$
\mu_{i}=\frac{m_{i}}{m_{b}+m_{i}}
$$

where $m_{b}$ and $m_{i}$ are the masses of the body and moving mass $i$, respectively.

The following assumptions are considered:

Assumption 1 The satellite body and the moving masses are rigid bodies.

Assumption 2 The moving mass is considered as a point mass.

Assumption 3 The moving masses' relative velocity, acceleration, and inertia have small magnitude with respect to the other quantity of the satellite.

Assumption 4 The satellite operates in a circular orbit and its angular velocity of the body-fixed coordinate system with respect to the inertial coordinate system is $\omega_{o}$.

\section{B. Aerodynamic Models}

The aerodynamic drag force acted on the satellite's center of pressure can be described as

$$
\boldsymbol{F}_{a}=-\frac{1}{2} \rho V^{2} C_{d} S_{p} \boldsymbol{v}
$$

where $\rho$ is the atmospheric density, $C_{d}$ is the drag coefficient, $v$ is the satellite velocity vector, $S_{p}$ is the total projected area of the flats. 
Considering the satellite consisting of several flat individual areas $S$ and individual normal vectors $\boldsymbol{n}_{k}$ in the body frame, the aerodynamic drag is given by

$$
\boldsymbol{F}_{a}=\sum_{k=1}^{6} \boldsymbol{F}_{a}^{k}=-\frac{1}{2} \rho V^{2} C_{d} S\left(\sum_{k=1}^{6}\left(\boldsymbol{n}_{k} \boldsymbol{v}\right) \operatorname{sgn}\left(\boldsymbol{n}_{k} \boldsymbol{v}\right)\right) \boldsymbol{v}
$$

where sgn is defined as the sign function. The term $S\left(\boldsymbol{n}_{k} \boldsymbol{v}\right)$ represents the projected area of each flat.

Similarly, the total aerodynamic drag moment acted on the satellite is also the sum of the aerodynamic drag moment on each flat:

$$
\boldsymbol{T}_{\text {aero }}=\sum_{k=1}^{6} \boldsymbol{T}_{\text {aero }}^{k}=\sum_{k=1}^{6} \boldsymbol{l}_{k} \times \boldsymbol{F}_{\text {aero }}^{k}
$$

where $\boldsymbol{l}_{k}=1 / 2 a \boldsymbol{n}_{k}$ denotes the arm of aerodynamic force.

For each pair of opposing flats, such as $\boldsymbol{n}_{1}$ and $\boldsymbol{n}_{4}$, the vector parts are the same:

$$
\left(n_{1} v\right)\left(n_{1} \times v\right)=\left(-n_{1} v\right)\left(-n_{1} \times v\right)=\left(n_{4} v\right)\left(n_{4} \times v\right)
$$

Thus, the total aerodynamic torque of the satellite is given by:

$$
\boldsymbol{T}_{a}=-\frac{1}{4} \rho V^{2} C_{d} a^{3} \sum_{k=1}^{3}\left(\boldsymbol{n}_{k} \boldsymbol{v}\right)\left(\boldsymbol{n}_{k} \times \boldsymbol{v}\right)
$$

It should be noted that when the center of mass of the system is coincided with the center of mass of the satellite body, the aerodynamic torque will not act on the satellite with moving mass control. That is, the satellite attitude is stable.

\section{Coupled Dynamics Modeling}

In this paper, we use quaternion $\boldsymbol{q}_{a}=\left[q_{0}, q_{1}, q_{2}, q_{3}\right]^{T}$ to describe the satellite kinematic model which be expressed by:

$$
\left[\begin{array}{c}
\dot{q}_{0} \\
\dot{\boldsymbol{q}}
\end{array}\right]=\frac{1}{2}\left[\begin{array}{c}
-\boldsymbol{q}^{T} \\
\boldsymbol{q}^{\times}+q_{0} \boldsymbol{I}_{3}
\end{array}\right] \omega_{e}
$$

where $\boldsymbol{q}=\left[q_{1}, q_{2}, q_{3}\right]^{T}$, and $\boldsymbol{I}_{3}$ is the identity matrix of proper dimension.

Let $\omega=\left[\omega_{x}, \omega_{y}, \omega_{z}\right]^{T}$ denote the the angular velocity of the satellite relative to the inertial frame in body-fixed frame. The satellite attitude dynamics can be given as

$$
I \dot{\omega}+\omega \times I \omega=T_{m}+T_{r}+d_{s}
$$


where $\boldsymbol{I}$ is the moment of inertia, $\boldsymbol{d}_{s}$ is the external disturbance, $\boldsymbol{T}_{m}$ is the magnetic moment, and $\boldsymbol{T}_{r}=\sum_{i=1}^{3} \boldsymbol{F}_{a} \times \mu_{i} \boldsymbol{r}_{i}$ is the aerodynamic moment generated from the aerodynamic force $\boldsymbol{F}_{a}$.

Since $\omega=\omega_{e}+\omega_{o}$, where $\omega_{e}$ is the angular velocity with respect to the geocentric inertial coordinate system, the attitude dynamics can be formulated as

$$
\boldsymbol{I} \dot{\boldsymbol{\omega}}_{e}=-\boldsymbol{I}\left(\omega_{o} \times \omega_{e}\right)-\left(\omega_{e}+\omega_{o}\right) \times\left[\boldsymbol{I}\left(\omega_{e}+\omega_{o}\right)\right]+\boldsymbol{T}_{m}+\boldsymbol{T}_{r}
$$

Consider that the following actual offsets

$$
\boldsymbol{r}_{m}=\sum_{i=1}^{3} \mu_{i} \boldsymbol{r}_{i}=\left[\begin{array}{l}
r_{x} \\
0 \\
0
\end{array}\right]+\left[\begin{array}{l}
0 \\
r_{y} \\
0
\end{array}\right]+\left[\begin{array}{l}
0 \\
0 \\
r_{z}
\end{array}\right]
$$

The offset of each moving mass is subject to the following saturation:

$$
r_{x, y, z}=\left\{\begin{array}{lr}
r_{x, y, z}^{\max } & \text { if } r_{x, y, z}^{c} \geq r_{x, y, z}^{\max } \\
r_{x, y, z}^{c} & \text { if }-r_{x, y, z}^{\max }<r_{x, y, z}^{c}<r_{x, y, z}^{\max } \\
-r_{x, y, z}^{\max } & \text { if } r_{x, y, z}^{c} \leq-r_{x, y, z}^{\max }
\end{array}\right.
$$

where $r_{x, y, z}^{c}$ is the command offset of each moving mass to be designed and $r_{x, y, z}^{\max }$ is the maximum offset of each moving mass.

The dynamics of relative motion is presented in the chief's LVLH frame as

$$
\ddot{\boldsymbol{p}}=\boldsymbol{A}_{1} \dot{\boldsymbol{p}}+\boldsymbol{A}_{2} \boldsymbol{p}+\boldsymbol{F}_{d}+\boldsymbol{f}_{d}
$$

where

$$
\boldsymbol{A}_{1}=\left[\begin{array}{ccc}
0 & 2 \omega_{r} & 0 \\
-2 \omega_{r} & 0 & 0 \\
0 & 0 & 0
\end{array}\right], \quad \boldsymbol{A}_{2}=\left[\begin{array}{ccc}
3 \omega_{r}^{2} & 0 & 0 \\
0 & 0 & 0 \\
0 & 0 & -\omega_{r}^{2}
\end{array}\right]
$$

and $\boldsymbol{B}_{1}=(1 / m) \boldsymbol{I}_{3}, \omega_{r}$ denotes the constant angular rate of the circular reference orbit. $\boldsymbol{F}_{d}=\boldsymbol{F}_{p}-\boldsymbol{F}_{c}$ is the differential aerodynamic control force, where $\boldsymbol{F}_{p}$ and $\boldsymbol{F}_{c}$ are the aerodynamic forces acting on the deputy satellite and chief satellite respectively. $f_{d}$ is the external disturbance. 


\section{Mechanism of attitude control}

In this section, we analyze the mechanism of the satellite with the moving mass control and quantify the relation between the attitude and the moving mass offsets. To this end, we first simplify the attitude dynamics to the linear equations and the following assumption is used for simplification.

Assumption 5 The attitude angles are small and their derivatives are negligible.

Based on the assumption, the product of the mass ratio and the attitude angle can be omitted. Then, the aerodynamic torque can be expressed in the body-fixed frame:

$$
\boldsymbol{T}_{r}=\boldsymbol{F}_{a}^{b} \times \sum \mu_{i} \boldsymbol{r}_{i}=-k_{d}\left[\begin{array}{ccc}
0 & \mu_{y} \theta & \mu_{z} \psi \\
-\mu_{x} \theta & 0 & \mu_{z} \\
-\mu_{x} \psi & -\mu_{y} & 0
\end{array}\right]\left[\begin{array}{c}
r_{x} \\
r_{y} \\
r_{z}
\end{array}\right] \approx k_{d}\left[\begin{array}{c}
0 \\
-\mu_{z} r_{z} \\
\mu_{y} r_{y}
\end{array}\right]
$$

where $\boldsymbol{F}_{a}^{b}=\boldsymbol{C}_{b s}(\phi, \theta, \psi) \boldsymbol{F}_{a}$ and $\boldsymbol{C}_{b s}$ is the rotation matrix transforming to the body-fixed frame from the inertial system frame. $\phi, \theta$, and $\psi$ denote the rolling, pitch, and yaw angle respectively.

Consequently, the simplified attitude dynamics without the magnetic control is given by

$$
\begin{aligned}
& I_{x} \ddot{\varphi}+\left(I_{y}-I_{z}-I_{x}\right) \omega_{o} \dot{\psi}+\left(I_{y}-I_{z}\right) \omega_{o}^{2} \varphi=0 \\
& I_{y} \ddot{\theta}=-k_{d} \mu_{z} r_{z} \\
& I_{z} \ddot{\psi}-\left(I_{y}-I_{z}-I_{x}\right) \omega_{o} \dot{\varphi}+\left(I_{y}-I_{x}\right) \omega_{o}^{2} \psi=k_{d} \mu_{y} r_{y}
\end{aligned}
$$

From the resulted dynamics, the offsets of the moving mass on the $z$-axis and the $y$-axis can control the pitch angle $\theta$ and yaw angle $\psi$, respectively. In particular, the first equation of Eq. 15 indicates that the moving mass is unable to control the roll angle. This is because the aerodynamic torque only exists in the plane perpendicular to the velocity direction of the satellite. Thus, the magnetic torquer is introduced to complete the attitude control system.

To further investigate the mechanism of the moving mass control, we consider the large angle maneuver and present 
the following nonlinear attitude dynamics:

$$
\begin{aligned}
I_{x} \dot{\omega}_{x}= & \left(I_{z}-I_{x}-I_{y}\right) c_{32} \omega_{o} \omega_{y}+\left(I_{x}-I_{y}+I_{z}\right) c_{22} \omega_{o} \omega_{z}+ \\
& \left(I_{y}-I_{z}\right)\left(\omega_{y} \omega_{z}+c_{22} c_{32} \omega_{o}^{2}\right)-k_{d} c_{31} \mu_{y} r_{y}+k_{d} c_{21} \mu_{z} r_{z} \\
I_{y} \dot{\omega}_{y}= & \left(I_{x}-I_{y}-I_{z}\right) c_{12} \omega_{o} \omega_{z}+\left(I_{y}-I_{z}+I_{x}\right) c_{32} \omega_{o} \omega_{x}+ \\
& \left(I_{z}-I_{x}\right)\left(\omega_{z} \omega_{x}+c_{32} c_{12} \omega_{o}^{2}\right)+k_{d} c_{31} \mu_{x} r_{x}-k_{d} c_{11} \mu_{z} r_{z} \\
I_{z} \dot{\omega}_{z}= & \left(I_{y}-I_{x}-I_{z}\right) c_{22} \omega_{o} \omega_{x}+\left(I_{z}-I_{x}+I_{y}\right) c_{12} \omega_{o} \omega_{y}+ \\
& \left(I_{x}-I_{y}\right)\left(\omega_{x} \omega_{y}+c_{12} c_{22} \omega_{o}^{2}\right)-k_{d} c_{21} \mu_{x} r_{x}+k_{d} c_{11} \mu_{y} r_{y}
\end{aligned}
$$

where $c_{i j}$ is the element of the rotation matrix $\boldsymbol{C}_{b s}$. Assume that the satellite maintain a stable attitude, the corresponding dynamics Eq. 16 becomes

$$
\begin{aligned}
& 0=\left(I_{y}-I_{z}\right)\left(c_{22} c_{32} \omega_{o}^{2}\right)-k_{d} c_{31} \mu_{y} r_{y}+k_{d} c_{12} \mu_{z} r_{z} \\
& 0=\left(I_{z}-I_{x}\right)\left(c_{32} c_{12} \omega_{o}^{2}\right)+k_{d} c_{31} \mu_{x} r_{x}-k_{d} c_{12} \mu_{z} r_{z} \\
& 0=\left(I_{x}-I_{y}\right)\left(c_{12} c_{22} \omega_{o}^{2}\right)-k_{d} c_{21} \mu_{x} r_{x}+k_{d} c_{11} \mu_{y} r_{z}
\end{aligned}
$$

It shows that the moving masses must shift to overcome the existence of the moment of inertia. Thus, we formulate Eq.(17) as follows:

$$
-k_{d} \underbrace{\left[\begin{array}{ccc}
0 & -c_{31} & c_{21} \\
c_{31} & 0 & -c_{11} \\
-c_{21} & c_{11} & 0
\end{array}\right]\left[\begin{array}{l}
\mu_{x} r_{x} \\
\mu_{y} r_{y} \\
\mu_{z} r_{z}
\end{array}\right]=\omega_{o}^{2}\left[\begin{array}{l}
\left(I_{y}-I_{z}\right)\left(c_{22} c_{32}\right) \\
\left(I_{z}-I_{x}\right)\left(c_{32} c_{12}\right) \\
\left(I_{x}-I_{y}\right)\left(c_{12} c_{22}\right)
\end{array}\right]}_{\boldsymbol{C}_{A}}
$$

Since the rank of $\boldsymbol{C}_{A}$ is 2 and the rank of the augmented matrix $\left(\boldsymbol{C}_{A} \mid \boldsymbol{C}_{B}\right)$ is 3, the attitude system is inconsistent. This result indicates that the attitude can be stabilized at an arbitrary status if and only if at least two of the three inertia are equal.

Based on the analysis of linear stability, we omit the equation of the roll axis because it is not affected by the motion of the moving masses. Then, it follows that

$$
\begin{aligned}
& \left(I_{z}-I_{x}\right)\left(c_{32} c_{12} \omega_{o}^{2}\right)=k_{d} c_{11} \mu_{z} r_{z} \\
& \left(I_{x}-I_{y}\right)\left(c_{12} c_{22} \omega_{o}^{2}\right)=-k_{d} c_{11} \mu_{y} r_{y}
\end{aligned}
$$

It can be clearly seen that the offset of the moving mass is proportional to the difference of the inertia. To determine 
the offset of the moving masses, Eq. 19 can be expanded as follow:

$$
\begin{aligned}
& r_{z}=\frac{\omega_{o}^{2}}{k_{d} \mu_{z}}\left(I_{z}-I_{x}\right)(-\tan \varphi \cos \psi+\cos \varphi \sin \theta \sin \psi) \tan \psi \\
& r_{y}=\frac{\omega_{o}^{2}}{k_{d} \mu_{y}}\left(I_{y}-I_{x}\right)(\cos \varphi \cos \psi+\sin \varphi \sin \theta \sin \psi) \tan \psi
\end{aligned}
$$

From Eq. [20], the yaw angle $\psi$ has a significant effect on the offset and the attitude angle due to the term $\tan \psi$. Particularly, when $\psi=0$, no matter how the pitch angle and the roll angle change, the offset $r_{y}$ and $r_{z}$ are zero. Furthermore, when $\psi$ is closed to $\pm \pi / 2$, the satellite can maintain a stable attitude only for $\theta=0$, otherwise $r_{y}$ and $r_{z}$ will be infinity.

To analyze the impact of the yaw angle on the stability range of the attitude, we conduct the maximum offsets of the moving mass with different yaw angles. First, the partial derivatives of $r_{z}$ with respect to $\theta$ and $\varphi$ are given by

$$
\begin{aligned}
& \frac{\partial r_{z}}{\partial \varphi}=\frac{\omega_{o}^{2}}{k_{d} \mu_{z}}\left(I_{z}-I_{x}\right)(-\cos \varphi \sin \psi-\sin \varphi \sin \theta \sin \psi \tan \psi) \\
& \frac{\partial r_{z}}{\partial \theta}=\frac{\omega_{o}^{2}}{k_{d} \mu_{z}}\left(I_{z}-I_{x}\right)(\cos \varphi \cos \theta \sin \psi \tan \psi)
\end{aligned}
$$

Let the above equations equal zero, one obtains $\varphi= \pm \pi / 2, \theta=0$ or $\varphi=-\pi / 2+\psi, \theta=\pi / 2$ or $\varphi=90^{\circ}-\psi, \theta=-90^{\circ}$. Then, the maximum $r_{z}$ is given by

$$
r_{z}^{\max }= \begin{cases}\mp \frac{\omega_{o}^{2}}{k_{d} \mu_{z}}\left(I_{z}-I_{x}\right) \sin \psi, & \varphi= \pm \pi / 2, \theta=0 \\ \frac{\omega_{o}^{2}}{k_{d} \mu_{z}}\left(I_{z}-I_{x}\right) \tan \psi, & \varphi=-\pi / 2+\psi, \theta=\pi / 2 \\ -\frac{\omega_{o}^{2}}{k_{d} \mu_{z}}\left(I_{z}-I_{x}\right) \tan \psi, & \varphi=\pi / 2-\psi, \theta=-\pi / 2\end{cases}
$$

The above equations can be formulated as follows:

$$
r_{z}^{\max }=\frac{\omega_{o}^{2}}{k_{d} \mu_{z}}\left|\left(I_{z}-I_{x}\right) \tan \psi\right|
$$

Subsequently, the partial derivatives of $r_{y}$ with respect to $\theta$ and $\varphi$ are given by

$$
\begin{aligned}
& \frac{\partial r_{y}}{\partial \varphi}=\frac{\omega_{o}^{2}}{k_{d} \mu_{y}}\left(I_{z}-I_{x}\right)(-\sin \varphi \sin \psi-\sin \varphi \sin \theta \sin \psi \tan \psi) \\
& \frac{\partial r_{y}}{\partial \theta}=\frac{\omega_{o}^{2}}{k_{d} \mu_{y}}\left(I_{z}-I_{x}\right)(\sin \varphi \cos \theta \sin \psi \tan \psi)
\end{aligned}
$$


Similarly, let the above equations equal zero, we obtain the maximum $r_{y}$ :

$$
r_{y}^{\max }=\frac{\omega_{o}^{2}}{k_{d} \mu_{y}}\left|\left(I_{y}-I_{x}\right) \tan \psi\right|
$$

From Eq. 23 and Eq. 25 , the yaw angles corresponded to $r_{z}^{\max }$ and $r_{y}^{\max }$ are derived as

$$
\begin{aligned}
& \psi_{z}=\arctan \left(\left|\frac{\mu_{z} r_{z}^{\max } k_{d}}{\left(I_{z}-I_{x}\right) \omega_{o}^{2}}\right|\right) \\
& \psi_{y}=\arctan \left(\left|\frac{\mu_{y} r_{y}^{\max } k_{d}}{\left(I_{y}-I_{x}\right) \omega_{o}^{2}}\right|\right)
\end{aligned}
$$

The above equations indicate that the maximum yaw angle depends on the limitation of the offset $r_{z}$ and $r_{y}$.

Remark 1 For the satellite with the moving mass control, the offset of the moving mass must be designed to overcome the moment of inertia, such that the desired attitude can be achieved. But the moment of inertia also is constrained from the attitude angle and the maximum offset of the moving mass. Therefore, if $|\psi| \leq \min \left\{\psi_{z}, \psi_{y}\right\}$, the desired attitude can be achieved by using Eq. (20). if $|\psi|>\min \left\{\psi_{z}, \psi_{y}\right\}$, the moving mass control is unable to stabilize the attitude.

\section{Formation control}

In this section, we design a formation control law for the proposed moving mass control of the orbital and attitude motions. The formation control problem is divided into two parts: 1) a Nash strategy taking disturbance into consideration is introduced to implement the desired differential accelerations; 2) an adaptive controller is investigated to generate the command offset of the moving mass.

\section{A. Orbit control}

The formation problem can be described as: design a command torque $F_{d}$ to implement three objectives: a desired relative configuration, a minimum aerodynamic consumption, and a minimum impact of disturbance. Therefore, the cost function for satellite $i$ is written as

$$
J_{i}=\frac{1}{2} \boldsymbol{e}_{r}^{T} \boldsymbol{Q} \boldsymbol{e}_{r}+\int_{0}^{t_{f}}\left[\boldsymbol{F}_{d i}^{T} \boldsymbol{R}_{u} \boldsymbol{F}_{d i}-\boldsymbol{f}_{d i}^{T} \boldsymbol{R}_{d} \boldsymbol{f}_{d i}\right] d t
$$

where the formation error is defined as $\boldsymbol{e}_{r}=\left[p_{i}-p_{l}-p_{d}, \dot{p}_{i}-\dot{p}_{l}-\delta\right]^{T}, \delta$ denotes desired relative distance vector, and the subscript $l$ denotes the leader satellite. $\boldsymbol{Q}, \boldsymbol{R}_{u}$, and $\boldsymbol{R}_{d}$ are the positive definite matrices.

Firstly, the Hamiltonian is introduced as follow:

$$
H_{i}=\frac{1}{2} \boldsymbol{F}_{d i}^{T} \boldsymbol{R}_{u} \boldsymbol{F}_{d i}-\frac{1}{2} \boldsymbol{f}_{d i}^{T} \boldsymbol{R}_{d} \boldsymbol{f}_{d i}+\lambda_{i}^{T}\left(\boldsymbol{A} \boldsymbol{x}_{i}+\boldsymbol{B} \boldsymbol{F}_{i}+\boldsymbol{C} \boldsymbol{f}_{d i}\right)
$$


where $\lambda_{i}$ is the costate vector, $\boldsymbol{x}_{i}=\left[\boldsymbol{p}_{i}, \dot{\boldsymbol{p}}_{i}\right]$, and

$$
\boldsymbol{A}=\left[\begin{array}{ll}
0_{3} & \boldsymbol{I}_{3} \\
\boldsymbol{A}_{1} & \boldsymbol{A}_{3}
\end{array}\right], \quad \boldsymbol{B}=\left[\begin{array}{l}
0_{3} \\
\boldsymbol{I}_{3}
\end{array}\right], \quad \boldsymbol{C}=\left[\begin{array}{l}
0_{3} \\
\boldsymbol{I}_{3}
\end{array}\right]
$$

Then, the following costate dynamics and stationarity condition for open-loop NE are

$$
\begin{gathered}
\dot{\lambda}_{i}=\frac{\partial H_{i}}{\partial \boldsymbol{x}_{i}}=-\boldsymbol{A}^{T} \lambda_{i} \\
\frac{\partial H_{i}}{\partial \boldsymbol{F}_{d i}}=\boldsymbol{R}_{u} \boldsymbol{F}_{i}+\boldsymbol{B}^{T} \lambda_{i}
\end{gathered}
$$

From the above equations, we have

$$
\begin{gathered}
\boldsymbol{F}_{d i}=-R_{u}^{-1} \Phi^{T}\left(t_{f}, t\right) \lambda_{i}\left(t_{f}\right) \\
f_{d i}=-R_{d}^{-1} \Phi^{T}\left(t_{f}, t\right) \lambda_{i}\left(t_{f}\right)
\end{gathered}
$$

where $\Phi^{T}\left(t_{f}, t\right)$ is the state-transition matrix of $\boldsymbol{A}$.

Substituting (32) and (33) into (13) and solving the resulting differential equation yields

$$
\boldsymbol{x}_{i}\left(t_{f}\right)=\Phi\left(t_{f}, 0\right) \boldsymbol{x}(0)-\int_{0}^{t_{f}} \Phi\left(t_{f}, \tau\right)\left[\boldsymbol{B} \boldsymbol{F}_{i}(\tau)+G d_{i}(\tau)\right] \mathrm{d} \tau
$$

Moreover, substituting the boundary condition $\boldsymbol{\lambda}_{i}\left(t_{f}\right)=\boldsymbol{Q} \boldsymbol{e}_{r}$ into 34 yields

$$
\boldsymbol{x}_{i}\left(t_{f}\right)=\Phi\left(t_{f}, 0\right) \boldsymbol{x}(0)-\int_{0}^{t_{f}} \Phi\left(t_{f}, \tau\right) \boldsymbol{B} \boldsymbol{R}_{u d} \boldsymbol{B}^{-1} \Phi^{T}\left(t_{f}, \tau\right) \mathrm{d} \tau \boldsymbol{Q}\left(\boldsymbol{x}_{i}\left(t_{f}\right)-\boldsymbol{x}_{l}-\boldsymbol{\delta}\right)
$$

where $\boldsymbol{R}_{u d}=\boldsymbol{R}_{u}^{-1}-\boldsymbol{R}_{d}^{-1}$.

It is straightforward to solve $\boldsymbol{x}_{i}\left(t_{f}\right)$ from the above equation as

$$
\boldsymbol{x}_{i}\left(t_{f}\right)=(\boldsymbol{I}+\overline{\boldsymbol{\Phi}})^{-1}\left[\Phi\left(t_{f}, 0\right) \boldsymbol{x}_{i}(0)+\bar{\Phi}\left(\boldsymbol{\delta}+\boldsymbol{x}_{l}\right)\right]
$$

where

$$
\bar{\Phi}=\int_{0}^{t_{f}} \Phi\left(t_{f}, \tau\right) B R_{u d} B^{-1} \Phi^{T}\left(t_{f}, \tau\right) \mathrm{d} \tau
$$

Substituting Eq. 36 and into Eq. 32 and Eq. 333 yields

$$
\boldsymbol{F}_{d i}^{*}=-\boldsymbol{R}_{u}^{-1} \Phi^{T}\left(t_{f}, t\right)\left\{(\boldsymbol{I}+\bar{\Phi})^{-1}\left[\Phi\left(t_{f}, t\right) \boldsymbol{x}_{i}(0)-\bar{\Phi} \boldsymbol{\delta}\right]-\boldsymbol{\delta}\right\}
$$




$$
\hat{\boldsymbol{f}}_{d i}=-\boldsymbol{R}_{d}^{-1} \Phi^{T}\left(t_{f}, t\right)\left\{(\boldsymbol{I}+\bar{\Phi})^{-1}\left[\Phi\left(t_{f}, t\right) \boldsymbol{x}_{i}(0)-\bar{\Phi} \boldsymbol{\delta}\right]-\boldsymbol{\delta}\right\}
$$

Since the control strategy $\left(\boldsymbol{F}_{d i}, \hat{\boldsymbol{f}}_{d i}\right)$ forms a saddle-point solution, its corresponding state $x_{i}$ can be considered as a "worst-case" state. furthermore, each satellite will find the optimal control that ensures the desired formation objective and rejects the disturbance under the individual cost function.

\section{B. Attitude control design}

It is obvious that implementation of moving mass control requires the command attitude which can be obtained from the results of the orbit control. Since there exists a mapping from the desired aerodynamic force $F_{d}$ to the command attitude, we construct the following nonlinear programming problem:

$$
\min J_{a}=k_{1} \theta_{d}^{2}+k_{2} \phi_{d}^{2}+k_{3} \psi_{d}^{2}
$$

subject to $\boldsymbol{F}_{d}=g\left(\phi_{d}, \theta_{d}, \psi\right)$ and $-\pi<\left\{\phi_{d}, \theta_{d}, \psi_{d}\right\} \leq \pi . k_{1}, k_{2}$, and $k_{3}$ are the weights of the cost function. Then, the optimization problem can be solved by MATLAB's fmincon function.

Consider the following Lyapunov quadratic function:

$$
V_{a}=K_{q} \boldsymbol{q}^{T} \boldsymbol{q}+k_{q}\left(q_{0}-1\right)^{2}+\frac{1}{2} \omega_{e}^{T} \boldsymbol{I} \omega_{e}+\frac{1}{2}\left(K_{I} \omega_{o}^{T} \omega_{o}-\omega_{o}^{T} I \omega_{o}\right)
$$

where $K_{q}$ and $K_{I} \geq \max \left\{I_{x}, I_{y}, I_{z}\right\}$ are positive constants. It is clear that the Lyapunov function is zero if and only if $q_{0}=1, \boldsymbol{q}=[0,0,0]^{T}$ and $\omega_{e}=[0,0,0]^{T}$.

The time derivative of $V_{a}$ is given by

$$
\dot{V}_{a}=2 K_{q} \boldsymbol{q}^{T} \dot{\boldsymbol{q}}+2 K_{q}(\boldsymbol{q}-1) \dot{\boldsymbol{q}}+\omega_{e}^{T} \boldsymbol{I} \dot{\omega}_{e}+K_{I} \omega_{o}^{T} \dot{\omega}_{o}-\boldsymbol{\omega}_{o}^{T} \boldsymbol{I} \dot{\boldsymbol{\omega}}_{o}
$$

We divide $\dot{V}$ into the following parts:

$$
\begin{gathered}
\dot{V}_{q}=2 k_{q} \boldsymbol{q}^{T} \dot{\boldsymbol{q}}+2 k_{q}\left(q_{0}-1\right) \dot{q}_{0} \\
\dot{V}_{\omega}=\boldsymbol{\omega}_{e}^{T} \boldsymbol{I} \dot{\boldsymbol{\omega}}_{e}+K_{I} \boldsymbol{\omega}_{o}^{T} \dot{\boldsymbol{\omega}}_{o}-\boldsymbol{\omega}_{o}^{T} I \dot{\boldsymbol{\omega}}_{o}
\end{gathered}
$$

By substituting Eq. 88 into $\dot{V}_{q}$, it becomes

$$
\dot{V}_{q}=K_{q} q^{T}\left(q^{\times}+q_{0} E_{3}\right) \omega_{e}-K_{q}\left(q_{0}-1\right) q^{T} \omega_{e}=K_{q} q^{T} \omega_{e}
$$


By substituting Eq. 10 into $\dot{V}_{\omega}$ and using mixed product operation, we have

$$
\begin{aligned}
\dot{V}_{\omega}= & -\omega_{e}^{T}\left\{I\left(\omega_{o}^{\times} \omega_{e}\right)+\left(\omega_{e}+\omega_{o}\right)^{\times}\left[I\left(\omega_{e}+\omega_{o}\right)\right]-T_{c}\right\}+ \\
& K_{I} \omega_{o}^{T}\left(\omega_{o}^{\times} \omega_{e}\right)+\omega_{o}^{T} I\left(\omega_{o}^{\times} \omega_{e}\right) \\
= & -\omega_{o}^{T} I\left(\omega_{o}^{\times} \omega_{e}\right)-\omega_{e}^{T} I\left(\omega_{o}^{\times} \omega_{e}\right)-\omega_{e}^{T} \omega_{o}^{\times}\left[I\left(\omega_{e}+\omega_{o}\right)\right]+\omega_{e}^{T} T_{c} \\
= & -\omega_{o}^{T} I\left(\omega_{o}^{\times} \omega_{e}\right)-\omega_{e}^{T} I\left(\omega_{o}^{\times} \omega_{e}\right)-\left(I \omega_{e}\right)^{T} \omega_{o}^{\times} \omega_{e}-\left(I \omega_{o}\right)^{T} \omega_{o}^{\times} \omega_{e}+\omega_{e}^{T} T_{c}
\end{aligned}
$$

Since the inertia matrix is symmetric, Eq. (46) becomes

$$
\dot{V}_{\omega}=\omega_{e}^{T} \boldsymbol{T}_{c}
$$

The control torque consists of the aerodynamic torque and magnetic torque:

$$
\boldsymbol{T}_{c}=\boldsymbol{T}_{m}+\boldsymbol{T}_{r}=\boldsymbol{F}_{a} \times \sum \mu_{i} \boldsymbol{r}_{i}-\boldsymbol{B}_{m}^{\times} \boldsymbol{M}_{B}
$$

where $\boldsymbol{B}_{m}$ is the column vector of the geomagnetic field and $\boldsymbol{L}_{m}$ is the column vector of the magnetic moment in the satellite body-fixed coordinate system.

The magnetic moment can be linearized as $\boldsymbol{L}_{m}=\boldsymbol{B}_{m}^{\times} /\|B\| r_{s}$, then the control torque is simplified to

$$
\boldsymbol{T}_{c}=\left(\boldsymbol{F}_{a}^{\times}-\boldsymbol{B}^{\times} \frac{\boldsymbol{B}^{\times}}{\|\boldsymbol{B}\|}\right) \boldsymbol{r}_{m}=\boldsymbol{P} \boldsymbol{r}_{m}
$$

Substituting Eq.445, Eq. (47) and Eq.449] into Eq.442), we have

$$
\dot{V}_{c}=\omega_{e}^{T}\left(K_{q} \boldsymbol{q}+\boldsymbol{P} \boldsymbol{r}_{m}\right)
$$

Then, the offset command of the moving mass is proposed by

$$
\boldsymbol{r}_{m}=-\boldsymbol{P}^{-1}\left(K_{q} \boldsymbol{q}+K_{\omega} \omega_{e}\right)
$$

Consequently, the time derivative of $V_{c}$ reduces to

$$
\dot{V}_{c}=-K_{\omega} \omega_{e}^{T} \omega_{e}
$$

Hence we can conclude that the attitude error converges to zero asymptotically. 


\section{Offset saturation}

To solve the offset saturation of the moving mass, an auxiliary variable is defined as

$$
\Delta_{s}=\boldsymbol{r}_{m}-\boldsymbol{r}_{m}^{c}
$$

and the attitude dynamics can be rearranged as follows:

$$
\boldsymbol{I} \dot{\omega}_{e}=\boldsymbol{f}_{a}+\boldsymbol{d}_{s}+\boldsymbol{T}_{c}
$$

Then, we define the total disturbance $\boldsymbol{d}_{a}=\boldsymbol{d}_{s}+\Delta_{s}$ and design an estimator to overcome it. The estimation error is defined as

$$
\boldsymbol{e}_{a}=\hat{\boldsymbol{d}}_{a}-\boldsymbol{d}_{a}+\boldsymbol{\beta}
$$

where $\beta$ is an additional function that improves the dynamics of the estimation errors.

From (IV.C), the time derivative of the estimation errors are given by

$$
\dot{\boldsymbol{e}}_{a}=\dot{\hat{\boldsymbol{d}}}_{a}+\frac{\partial \boldsymbol{\beta}}{\partial \boldsymbol{q}} \dot{\boldsymbol{q}}_{a}+\frac{\partial \boldsymbol{\beta}}{\partial \omega_{e}}\left[\boldsymbol{I}^{-1}\left(\boldsymbol{f}_{a}+\boldsymbol{d}_{a}+\boldsymbol{T}_{c}\right)\right]
$$

Then, we choose the following update law:

$$
\dot{\hat{\boldsymbol{d}}}_{a}=-\frac{\partial \boldsymbol{\beta}}{\partial \boldsymbol{q}} \dot{\boldsymbol{q}}_{a}-\frac{\partial \boldsymbol{\beta}}{\partial \omega_{e}}\left[\boldsymbol{I}^{-1}\left(\boldsymbol{f}_{a}+\hat{\boldsymbol{d}}_{a}+\boldsymbol{\beta}+\boldsymbol{T}_{c}\right)\right]
$$

Substituting 57 into 56 yields:

$$
\dot{\boldsymbol{e}}_{a}=-\frac{\partial \boldsymbol{\beta}}{\partial \omega_{e}} \boldsymbol{e}_{a}
$$

It can be seen that the function $\beta$ plays an important role of keeping the dynamic of $\boldsymbol{e}_{a}$ stable.

Considering the following Lyapunov function

$$
V_{d}=\frac{1}{2} \boldsymbol{e}_{a}^{T} \boldsymbol{e}_{a}
$$

The time derivative of $V_{d}$ is

$$
\dot{V}_{d}=-\boldsymbol{e}_{a}^{T} \frac{\partial \boldsymbol{\beta}^{T}}{\partial \omega} \boldsymbol{e}_{a}
$$

If we select $\partial \boldsymbol{\beta} / \partial \omega=\gamma_{a}$, then $\dot{V}_{d}=-\gamma_{a} \boldsymbol{e}_{a}^{2} \leq 0$. Hence, the estimation error $e_{a}$ converges to zero. It should be noted that the estimation convergence performance can be improved by the estimation gain $\gamma_{a}$. 


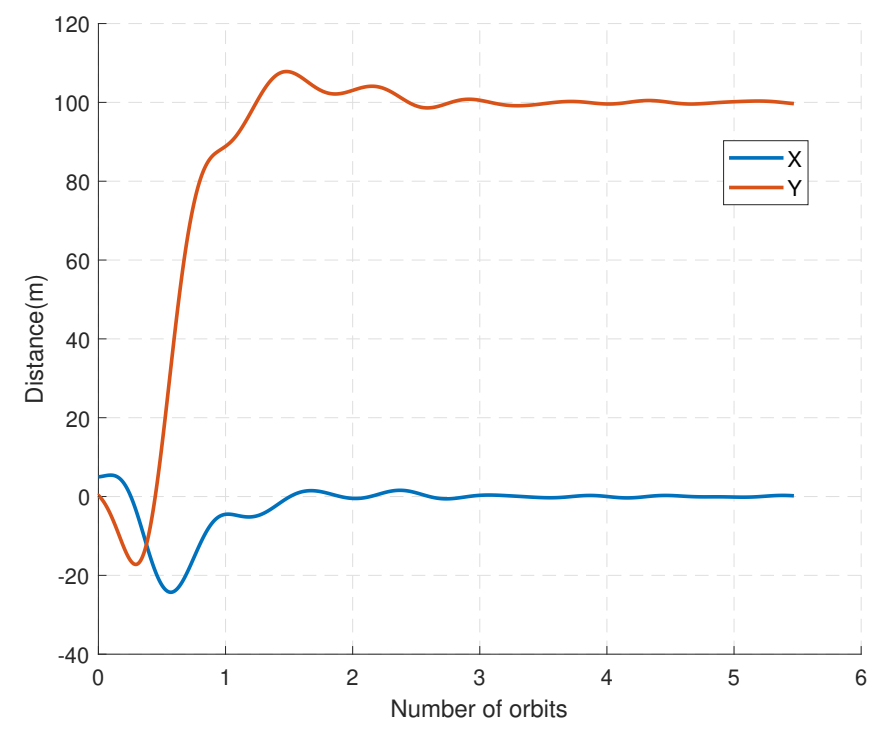

Fig. 2 Formation error

With the estimation Eq. 58 and the auxiliary variable, the complete attitude control law is given by

$$
\boldsymbol{r}_{m}=-\boldsymbol{P}^{-1}\left(K_{q} \boldsymbol{q}+K_{\omega} \boldsymbol{\omega}_{e}\right)-\hat{\boldsymbol{d}}_{a}
$$

\section{Simulation}

In this section, a two satellites formation scenario is presented to verify the moving mass control method. The chief satellite operates in an circular orbit with the orbit radius of $6728 \mathrm{~km}$. The atmospheric density is $2.803 \times 10^{-12} \mathrm{~kg} / \mathrm{m}^{3}$ and the corresponding drag coefficient $C_{d}=2.2$. The initial and desired formation error are given by

$$
\begin{aligned}
& E_{0}=[10,0,0]^{T} \mathrm{~m} \\
& E_{f}=[0,100,0]^{T} \mathrm{~m}
\end{aligned}
$$

The side-length of the satellite is $0.3 \mathrm{~m}$ and the moving mass takes $8 \%$ of the $1 \mathrm{~kg}$ satellite and have a $100 \mathrm{~mm}$ feasible offset. The inertia matrix of the moving mass satellite is $\operatorname{diag}\{0.011,0.0138,0.0043\} \mathrm{kg} \cdot \mathrm{m}^{2}$. Fig 2 presents the position error between the chief and deputy satellite. It can be seen that the desired formation can be achieved in less than 3 orbits.

As shown in Fig 3 the attitudes change to generate the aerodynamic torques and converge to zero finally. The offsets of the moving masses and the magnetic torques are shown in Fig 4 and Fig 5 As expected, when the system has achieved the desired formation, the moving masses stay at the origin because the attitude stabilization is achieved. 


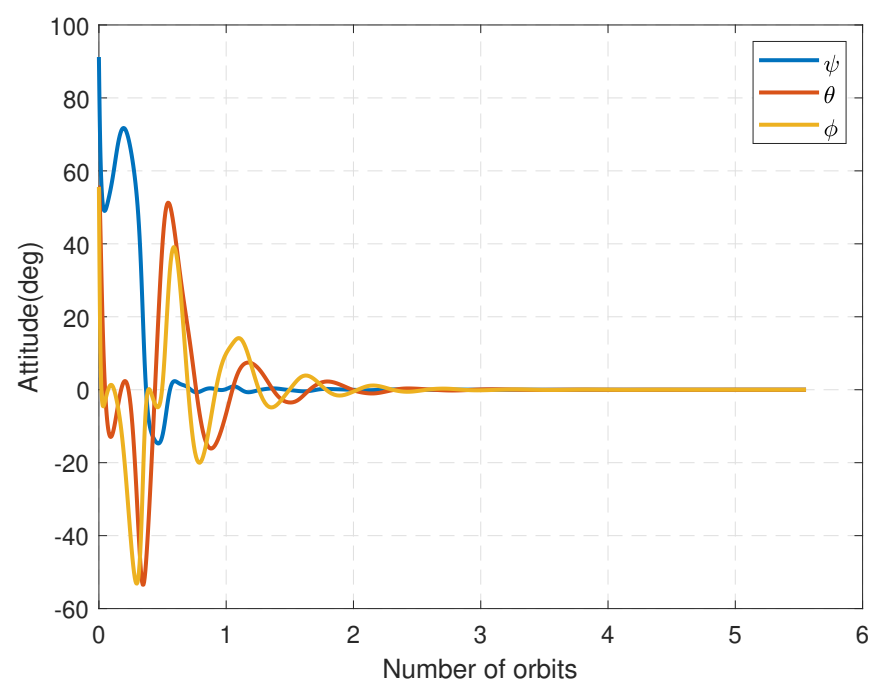

Fig. 3 Attitude

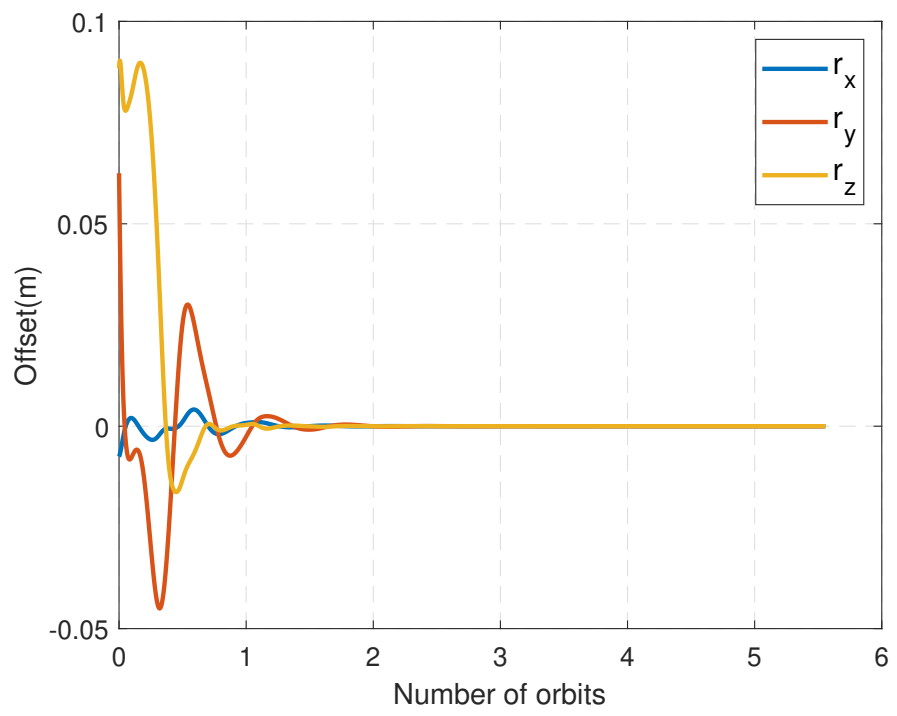

Fig. 4 Offsets of the moving masses 


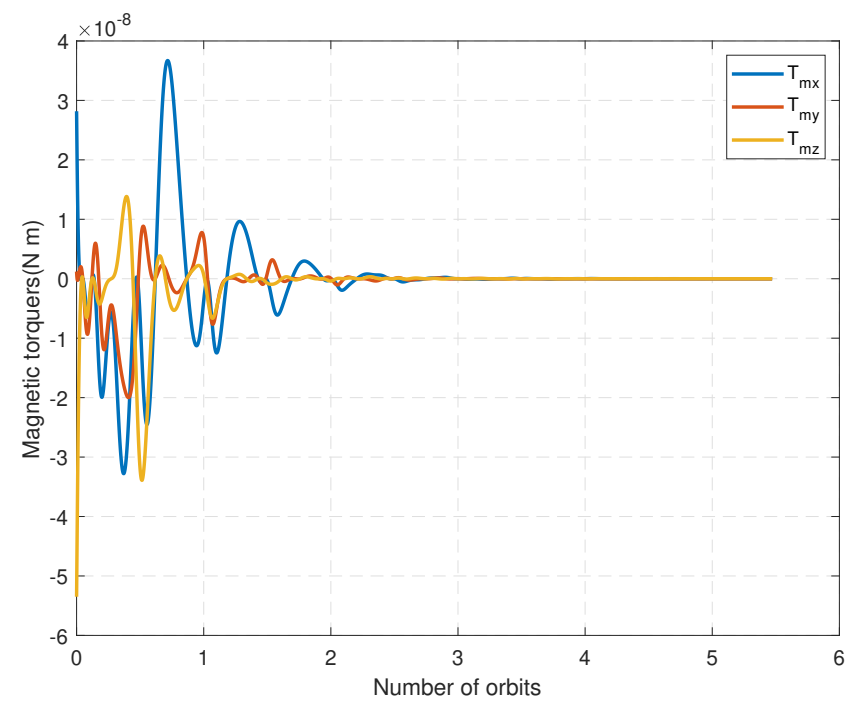

Fig. 5 Magnetic torquers's torque

\section{Conclusion}

This paper proposes a methodology to perform formation control using moving masses. In particular, by investigating the mechanism of the proposed moving mass control scheme, it shows that the limitation of the offset of the moving mass determines the attitude maneuver capability, especially on the yaw angle. The analysis results present the quantitative relation between the attitude and the offsets of moving masses, and provide the reference for designing the mass ratio and the offset ranges. Then, a control strategy based Nash game is presented to realize the formation configuration. Particularly, the worst-case Nash is applied to overcome the effect of unknown disturbances. In view of the limitation of actuators, a compensation observer is designed to deal with the the offset saturation of moving masses. Simulation results show that the use of moving mass control based on the atmospheric forces is particularly advantageous for LEO satellite formation.

\section{Acknowledgments}

This work is supported in part by the National Natural Science Foundation of China (Grant No. 11802268) and by the Key Lab Foundation of Space Intelligent Control Systems (Grant No. 6142208200306).

\section{Data availability}

The datasets generated during and/or analyzed during the current study are available from the corresponding author on reasonable request. 


\section{Declarations}

Conflict of interest The authors declare that they have no conflict of interest.

\section{References}

[1] Leonard, C. L., Hollister, W. M., and Bergmann, E. V., “Orbital formationkeeping with differential drag,” Journal of Guidance Control, and Dynamics, Vol. 10, No. 1, 2012, pp. 755-765.

[2] Harris, M. W., and Acikmese, B., "Minimum Time Rendezvous of Multiple Spacecraft Using Differential Drag," Journal of Guidance, Control, and Dynamics, Vol. 2, No. 2, 2015, pp. 365-373.

[3] Ben-Yaacov, O., and Gurfil, P., “Long-Term Cluster Flight of Multiple Satellites Using Differential Drag,” Journal of Guidance Control, and Dynamics, Vol. 36, No. 6, 2013, pp. 1731-1740.

[4] Harris, A. T., Petersen, C. D., and Schaub, H., "Linear Coupled Attitude-Orbit Control Through Aerodynamic Drag," Journal of Guidance, Control, and Dynamics, Vol. 43, No. 1, 2019, pp. 1-10.

[5] Horsley, M., Nikolaev, S., and Pertica, A., "Small Satellite Rendezvous Using Differential Lift and Drag," Journal of guidance, control, and dynamics, Vol. 36, No. 2, 2013, pp. 445-453.

[6] Janssens, F. L., and Ha, J., "Stability of Spinning Satellite Under Axial Thrust, Internal Mass Motion, and Damping," Journal of Guidance Control and Dynamics, Vol. 38, No. 4, 2015, pp. 761-771.

[7] White, J. E., and Robinett, R., "Principal axis misalignment control for deconing of spinning spacecraft," Journal of Guidance, Control, and Dynamics, 1994.

[8] Wie, and Bong, "Solar Sail Attitude Control and Dynamics, Part 1," Journal of Guidance Control and Dynamics, Vol. 27, No. 4, 2004, pp. 536-544.

[9] Kumar, K., "Attitude Control of Miniature Satellites Using Movable Masses," SpaceOps 2010 Conference Delivering on the Dream Hosted by NASA Marshall Space Flight Center and Organized by AIAA, 2013.

[10] “Automatic Mass Balancing of a Spacecraft Three-Axis Simulator: Analysis and Experimentation,” Journal of Guidance, Control, and Dynamics, 2014.

[11] Virgili-Llop, J., Polat, H. C., and Romano, M., "Using Shifting Masses to Reject Aerodynamic Perturbations and to Maintain a Stable Attitude in Very Low Earth Orbit,” 26th AAS/AIAA Space Flight Mechanics Meeting, 2016.

[12] Chesi, S., Qi, G., and Romano, M., "Aerodynamic Three-Axis Attitude Stabilization of a Spacecraft by Center-of-Mass Shifting," Journal of Guidance Control and Dynamics, Vol. 40, No. 7, 2017, pp. 1613-1626.

[13] Ross, and Michael, I., "Mechanism for Precision Orbit Control with Applications to Formation Keeping," Journal of Guidance Control and Dynamics, Vol. 25, No. 4, 2015, pp. págs. 818-819. 